\title{
Is active targeting of brain metastases of breast cancer superior to passive targeting?
}

\author{
Nermeen H. Kamal' ${ }^{1, *}$, Fatema El-Amrawy ${ }^{2, *}$, Heba Abdullah $\mathrm{Ali}^{3}$, Ivan Edafiogho ${ }^{4}$, Mohamed Ismail \\ Nounou $^{4}$ \\ 'Department of Pharmaceutics, College of pharmacy, Arab Academy for Science, Technology and Maritime Transport \\ (AASTMT), Alexandria 1029, Egypt. \\ ${ }^{2}$ Biomedical Informatics and Medical Statistics Department, Medical Research Institute (MRI), Alexandria University, Alexandria \\ 21561, Egypt. \\ ${ }^{3}$ Department of Pharmaceutics and Industrial Pharmacy, College of Pharmacy for Girls, Al Azhar University, Cairo 11651, Egypt. \\ ${ }^{4}$ Department of Pharmaceutical Sciences (DPS), School of Pharmacy and Physician Assistant Studies (SOPPAS), University of \\ Saint Joseph (USJ), Hartford, CT 06103, USA. \\ *Both authors contributed equally to the manuscript and are both considered first authors
}

Correspondence to: Dr. Mohamed Ismail Nounou, Department of Pharmaceutical Sciences (DPS), School of Pharmacy and Physician Assistant Studies (SOPPAS), University of Saint Joseph (USJ), Hartford, CT 06103, USA. E-mail: nounou@usj.edu

How to cite this article: Kamal NH, El-Amrawy F, Ali HA, Edafiogho I, Nounou MI. Is active targeting of brain metastases of breast cancer superior to passive targeting? J Cancer Metastasis Treat2019;5:11. http://dx.doi.org/10.20517/2394-4722.2018.89

Received: 7 Dec 2018 First Decision: 29 Dec 2018 Revised: 7 Jan 2019 Accepted: 22 Jan 2019 Published: 20 Feb 2019

Science Editor: William P. Schiemann Copy Editor: Cai-Hong Wang Production Editor: Huan-Liang Wu

\begin{abstract}
Brain metastasis is a major cause of death in patients with solid cancers. Breast cancer cells have high tendency to migrate towards brain. Cancer cells within brain are characterized by severe aggressiveness and inaccessibility. Currently, breast cancer and its metastasis are the second leading cause of death among women. Tumor microenvironment and blood brain barrier (BBB) represent great obstacles in targeting breast cancer and its metastasis. Chemotherapy is a safer treatment modality for brain metastasis compared with risky surgical resection and brain radiotherapy. Unfortunately, conventional chemotherapy lack penetration of BBB and suffer from multiple resistance mechanisms. Current treatment technologies for brain metastases of breast cancer have limited long-term success and numerous side effects, illustrating the urgent need for novel smart strategies. Various novel drug entities and nanosystems have been employed to improve diagnosis and targeted treatment of breast cancer and its metastasis. Immunotherapy agents and small tyrosine kinase inhibitors have been shown to reduce tumor size and increase survival in patients with breast cancer, but still poorly penetrate BBB. Tailored sized nanoparticles to some extent crossed brain tumor barrier and enhanced drug accumulation in tumors by taking advantage of enhanced permeability and retention. Furthermore, various active targeting strategies have been adopted to improve accessibility to brain malignancies. Therefore, to achieve enhanced antitumor therapy against
\end{abstract}

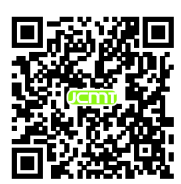


breast cancer and its brain metastasis, multi-talented delivery systems are urgently needed for optimal treatment. This review focuses on the various active and passive targeting technologies for the treatment of breast cancer brain metastases in the past decade. A comprehensive summary and examples along with pros and cons of each system will be discussed. Different treatment modalities and nanotechnology facilities will be demonstrated to aid in designing the optimal smart, safe, targeted and effective systems to combat brain metastases of breast cancer.

Keywords: Active targeting, passive targeting, brain metastasis, breast cancer

\section{INTRODUCTION}

Despite the recent pharmaceutical and medical advances in treatment and diagnosis of different carcinogenic tissues and their metastases, brain and central nervous system (CNS) primary and metastatic tumors remain a tough hurdle to overcome due to physiological and anatomical barriers represented by blood brain barrier (BBB) and the aggressiveness and continuous adaptive evaluation of this tumor tissue.

As systemic therapy of metastatic breast cancer advances, survival rates of patients are enhanced. On the other side, the incidence of brain and CNS complications increases ${ }^{[1]}$. In historical series, the risk of developing brain metastasis has been estimated among women with stage IV breast cancer to range from $10 \%$ to $16 \%$ among living making it the second most common cause of metastatic brain tumor after lung cancer $(10 \%-25 \%)^{[2-4]}$. The development of breast cancer brain metastases (BCBM) tends to vary by the subtype. Forty-six percent of patients with advanced triple negative breast cancer, and up to thirty-seven percent of patients with HER2-positive breast cancer relapse due to intracranial metastases, despite control of the peripheral tumors ${ }^{[5-8]}$.

Treatment options are limited and usually involve multimodality approaches that include surgery, radiotherapy, radiosurgery, and rarely systemic therapy, depending on the number of CNS lesions ${ }^{[9,10]}$. The unpredictable extent of therapeutics that can reach tumor vasculature of BCBM has been the challenge in developing effective treatments for these patients ${ }^{[11,12]}$. BBB has always been a challenge to drug delivery to brain tissues ${ }^{[13-16]}$. However, during brain metastases, the structure and integrity are altered forming a "Blood Brain Tumor Barrier" (BBTB) ${ }^{[17]}$. The leaky structure of BBTB is an appealing strategy to target brain tumors $^{[18]}$. Approaches to deliver drugs into the brain are being extensively studied [Figure 1].

Beside the common technologies including viral vectors and nanoparticles, novel non-invasive techniques such as ultrasound alteration and magnetic stimulation have been studied to temporally open the BBB to enhance brain drug uptake. In this review, we discuss passive and active targeting BCBMs.

\section{BBB AND DRUG DELIVERY}

The $\mathrm{BBB}$, as a morphologic and physiologic barrier, protects the brain tissue from the peripheral vasculature, protecting normal brain function by impeding most compounds from transiting from the blood to the brain $^{[13,16,18]}$. There is a common misconception about the nature of BBB as a protective barrier or sac protecting the brain underneath it. Perhaps, the term BBB was the main reason of such misconception. The BBB is mainly a fortified special vascular structure in the brain different from the common vascular structure in the rest of the body. Mainly, the brain capillary endothelial cells (BCECs) form the BBB, besides other cell types such as pericytes, astrocytes, and neuronal cells; playing an important role in the function of the $\mathrm{BBB}^{[19]}$. BCEC have tight junctions that prevent paracellular transport of small and large (water soluble) compounds from the circulation to the brain except for some very small or gaseous molecules, such as water and carbon dioxide ${ }^{[17,19,20]}$. The components of the BBB continuously adapt in response to various physiological changes in the brain ${ }^{[21]}$ [Figure 1]. 


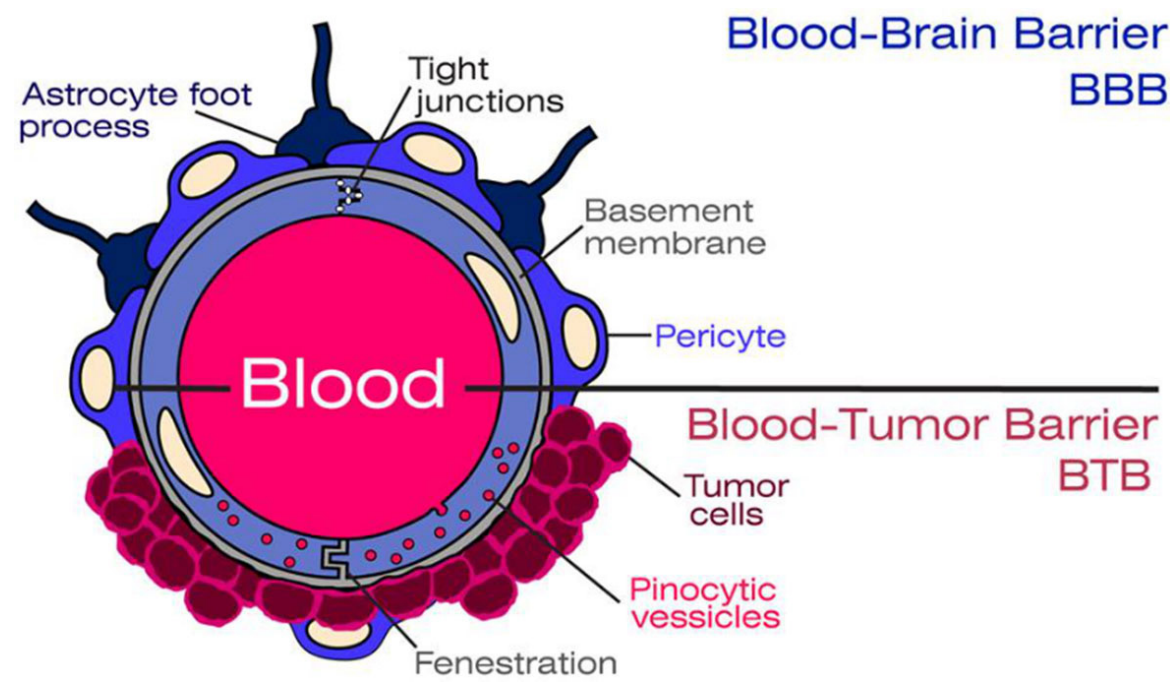

Figure 1. The vascular and structural components of the blood brain barrier (BBB) and the blood brain tumor barrier

Similarly, BCECs restrict drug distribution into the brain by limiting drug diffusion from blood to brain. In addition to these physical barriers, cells, like pericytes, and astrocyte foot processes, express several drug metabolizing enzymes that inactivate drugs reducing the distribution of active drugs to the brain. Thus, complicating barrier mechanisms \& the presence of both drug-uptake and drug efflux mechanisms such as multidrug transporters including P-glycoprotein (ABCB1/MDR1) and ABCG2. Some compounds pass the BBB by passive non-saturable diffusion, some by active transport; others are actively excluded, and still, others enter through endocytosis ${ }^{[12]}$.

\section{BRAIN METASTASES OF BREAST CANCER}

The occurrence of brain metastases depends on how breast cancer cells adapt to and survive in foreign environments $^{[22]}$. Specific homing molecules mediate organ-specific metastasis formation on the heterogeneous tumor cell surface ${ }^{[23]}$. Investigating throughout the metastatic cascade is one area of research that can lead to the development of effective treatment.

Research has shown that at the time of initial diagnosis of primary cancer, circulating tumor cells can be found in the bloodstream of patients ${ }^{[24]}$. Breast cancer stem cells are characterized by the ability to self-renew along with their high level of resistance to radiation and chemotherapeutic agents ${ }^{[2]}$. After detachment of tumor cells from the primary lesion, invasion into the CNS and bypassing the BBB occur, followed by sustained proliferation towards the formation of brain metastases ${ }^{[2,27]}$. Studies have suggested that brain metastases originate from cells as a primary mass or lymph nodes or from other visceral metastases ${ }^{[28]}$.

Disruption of the $\mathrm{BBB}$ was shown to involve mediators of extravasation through non-fenestrated capillaries. A number of mediators have been identified to assist breast cancer cells crossing the BBB, including cyclooxygenase-2, heparin-binding epidermal growth factor-like growth factor, and the $\alpha 2,6$-sialyltransferase ST6GALNAC5 $5^{[29-34]}$.

The recently identified extracellular (circulating or exosomal) miRNAs were shown to mediate the ability of metastatic breast cancer cells to target the distant brain endothelium and vasculature ${ }^{[35]}$. The metastatic BC cells in brain express high levels of anti-plasminogen activator serpins as a shield to escape from the reaction of brain stroma, conferring the adherence of infiltrating breast cancer cells to the surface of capillaries and the growth on the vasculature ${ }^{[36]}$. 


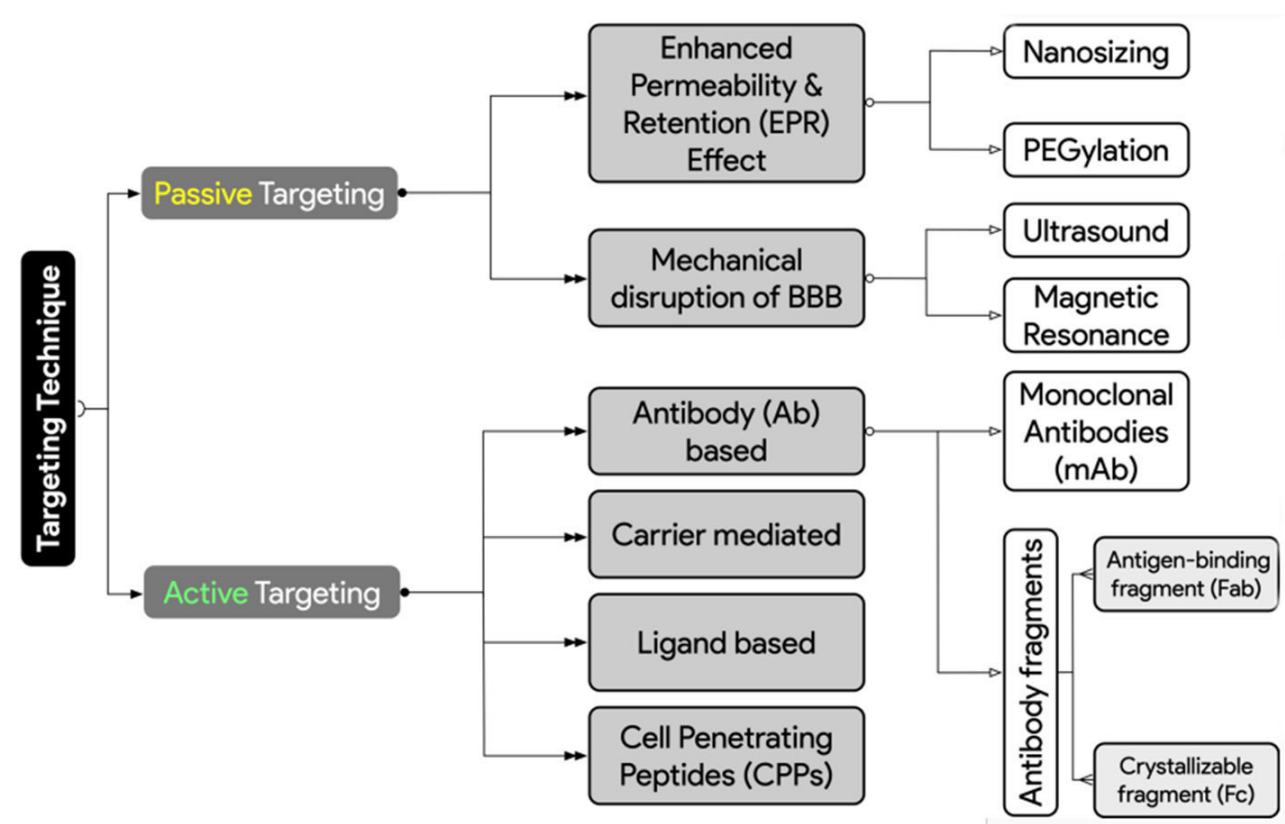

Figure 2. Different adopted strategies for actively and passively targeting brain metastases of breast cancer to overcome the blood brain barrier (BBB) hurdles

Brain metastases of breast cancer are also subject to the limited permeability characteristics of the BBB in-spite of the formation of the BBTB with altered integrity. MRI data have shown that not all brain metastases display elevated BBTB permeability ${ }^{[37]}$. The changes in BBTB vascular permeability are usually not homogenous throughout the lesion ${ }^{[38,39]}$. It was noticed that brain metastases from HER2+ breast cancers infiltrate brain tissue crossing the endothelial cells without disrupting the BBB, unlike the brain metastases from triple negative or basal-type breast cancers that often disrupt the $\mathrm{BBB}^{[8,40]}$ [Figure 1].

\section{TARGETING BRAIN METASTASES}

Although, there are no FDA-approved systemic treatments for BCBM to date ${ }^{[41]}$, patents and studies in the past years have shown promising progress and well-established techniques to overcome the BBB/ $\mathrm{BBTB}^{[42]}$. There are two general strategies adopted to facilitate crossing the BBB; invasive and non-invasive techniques ${ }^{[43]}$. The invasive techniques rely primarily on disrupting the BBB integrity by direct intracranial drug delivery through intracerebroventricular, intracerebral or intrathecal administration, osmotic pumps or biochemical means ${ }^{[43]}$. But all these approaches are severely limited by poor distribution into brain parenchyma ${ }^{[44]}$. On the other hand, non-invasive methods include drug moieties modifications through the transformation of the drug into lipophilic analogs, prodrugs, chemical drug delivery, carrier-mediated drug delivery, receptor/vector-mediated drug delivery and intranasal drug delivery ${ }^{[43,45]}$.

Diffusion of substances into the brain can be divided into paracellular and transcellular. Generally, BBB targeting strategies can be categorized into passive (transcellular lipophilic pathway) and active targeting (mainly; transcytosis) ${ }^{[46]}$. Figure 2 summarizes the targeting techniques covered in this article.

\section{Passive targeting}

Enhanced permeability and retention phenomenon

Passive targeting depends mainly on the preferential accumulation of drug molecules into tumor cells $\mathrm{s}^{[47]}$. Enhanced permeability and retention (EPR) phenomenon based on the nanometer size range of the nanoparticles and two fundamental characteristics of the neoplastic tissues, namely, the leaky vasculature and impaired lymphatic drainage. EPR was first described by Maeda and Matsumura ${ }^{[48]}$. The selective high 
local concentration of nanosized anticancer drugs in tumor tissues enhances the therapeutic effect with minimal side effects in both preclinical and clinical settings ${ }^{[49]}$.

Various important factors such as circulation time, targeting and the capability to overcome BBB are heavily reliant on the shape, size and the surface area of these particles. In passive diffusion, lipid solubility, molecular weight, and the presence of tight junctions greatly affect the degree of permeation ${ }^{[0,51]}$.

Conventionally, a particle must be at least $10 \mathrm{~nm}$ in diameter to avoid clearance by first-pass renal filtration and the optimal size range of 100-180 nm will ensure longer circulation time, increased accumulation within the tumor mass and lower renal clearance ${ }^{[52,53]}$. Moreover, surface characteristics also play a very important role in determining the extent of internalization of these nanoparticles into cells. Relatively, the surface can be modified by the polymer composition, thus governing an extra amount of hydrophobicity or hydrophilicity to these particles ${ }^{[51]}$. Supplementary Table 1 illustrates some of the papers using passive targeting technology in targeting BCBM.

\section{Nanotechnology as a tool for targeting EPR}

Nanotechnology paved the road towards safer and more likely effective cancer treatment strategies that can overcome some of the hurdles for drug delivery. Nano-medicines primarily aim to improve the circulation time of the conjugated or entrapped (chemo-) therapeutic drugs ${ }^{[54]}$. Examples of nanoparticles commonly used include: polymeric particles, polymeric micelles, dendrimers, and liposomes. Nanoparticles represent versatile tools to encapsulate various types of drugs, either hydrophilic or hydrophobic moieties altering their physicochemical parameters and pharmacokinetics profile ${ }^{[55]}$.

Beside increasing drug-site contact time, some polymers used in nanoparticles formulations such as polylactic-co-glycolic acid (PLGA) have high cell adhesion property, increasing the drug concentration gradient at the adhesion site by longer drug carrier contact time with the targeted cells ${ }^{[56]}$.

However, nanoparticles can show some serious adverse effects ${ }^{[57]}$. Adverse effects of nanoparticles depend on individual factors such as genetics, existing disease conditions, exposure, nanoparticle chemistry, size, shape, agglomeration state, and electromagnetic properties ${ }^{[57]}$. The key to understanding the toxicity of nanoparticles is their size; nanoparticles are smaller than cells and cellular organelles, which allow them to penetrate these biological structures, disrupting their normal function ${ }^{[57]}$. Examples of toxic effects include tissue inflammation, and altered cellular redox balance toward oxidation, causing abnormal function or cell death ${ }^{[57]}$. Furthermore, a major drawback is the difficulty in scaling up the formulation and its transformation to clinical use, due to high cost and instability during storage. The NP materials must be biocompatible and safe when administered ${ }^{[58]}$.

\section{Lipsomes}

Liposomal (Liposome: lipid vesicle in Latin) nanocarrier systems are vesicular lipid bilayer colloidal spheres formed by self-assembly ${ }^{[59]}$. Without the use of surface modification by a hydrophilic polymer such as polyethylene glycol (PEG), the biological half-lives of liposomes are very short due to several factors including the tendency of the liposome to exchange lipid materials with cell membranes and their uptake by phagocytes. Mohammad et al. ${ }^{[60]}$ noticed increased survival rates in animals treated with liposomal irinotecan (nal-IRI) that sustained the accumulation of irinotecan and its active metabolite in the brain metastases lesions, unlike non-liposomal irinotecan whose distribution in the CNS was hampered by BBTB and various efflux proteins. The size of nal-IRI was between 100-110 nm, which enhanced its preferential accumulation within tumor due to EPR through the leaky vasculature of the tumor ${ }^{[60]}$. Drawbacks of liposomes involved their rapid uptake by the Reticulo-Endothelial System (RES) and consequent removal from circulating blood. 
PEGylation

The main pharmacokinetic outcomes of PEGylation include: changes occurring in overall circulation lifespan, tissue distribution pattern, and elimination pathway of the parent drug/particle ${ }^{[61]}$. PEG mainly protects these particles from being phagocytosed by natural particle eliminating mechanisms, mainly organs of the RES ${ }^{[62]}$.

Pegylated nanoparticles are characterized by hydrophilic surfaces that circumvent opsonization and decrease their clearance by macrophage, which results in prolonged circulation ${ }^{[51]}$. PEGylation has been shown to alter the pharmacokinetics of doxorubicin considerably; the total clearance was significantly reduced ${ }^{[63]}$. In the early 1990's, PEGylated polymeric vesicles were introduced by Yokoyama et al. ${ }^{[64]}$, which represented an important milestone in the synthesis of long-circulating liposomal formulations (STEALTH ${ }^{\bullet}$ liposomes). Liposomal formulations are the first novel controllable carrier systems to be sold in the market for cancer (Doxil ${ }^{\odot}$, PEGylated liposomal formulation encapsulating doxorubicin(PLD) ${ }^{[65-67]}$. PLD showed reduced cardiotoxicity and prolonged activity.

Anders et al. ${ }^{[68]}$ encapsulated doxorubicin in pegylated liposomes (PLD) that achieved 20 folds higher concentration of doxorubicin within intracranial tumors compared to non-liposomal doxorubicin, whose distribution was compromised by BBTB. Moreover, co-administration of PLD and BBB permeable ABT888, an inhibitor of a poly (ADP-ribose) polymerase, showed better survival as compared to non-liposomal doxorubicin and ABT-888.

Nektar ${ }^{\mathrm{TM}}$ Therapeutics has several patents on PEGylation bioconjugation aiming to modify the pharmacokinetic profile ${ }^{[69-75]}$. Adkins et al. ${ }^{[76]}$ formulated NKTR-102, an Irinotecan-PEG conjugate linked with a hydrolysable ester bond, whose polymer moiety resulted in prolonged circulation and subsequently increased tumor localization. The innovative nature of this PEGylated system is the adoption of the threedimensional (3D) branching technology in the PEGylation to provide superior pharmacokinetics and pharmacokinetic parameters. The 3D propriety Nektar PEG technology offers weeks of half-lives compared to days in conventional $\mathrm{PEG}^{[76]}$. The preferential accumulation of NKTR-102 within brain metastases via EPR, its reduced clearance and ability to escape P-glycoprotein mediated efflux resulted in continuous release of the active metabolite $\mathrm{SN} 38$ and better therapeutic efficacy.

Nektar ${ }^{\mathrm{TM}}$ Therapeutics is not the only pharmaceutical company relying on innovative PEGylation technologies for passively targeting brain metastases of breast cancer. Other companies, such as 2-BBB, is investing heavily on the use of PEGylation bioconjugated with liposomal vesicular carrier systems to passively target brain metastases of breast cancer ${ }^{[77]}$. BBB adopts a propriety G-Technology ${ }^{\varnothing}$ for targeting the brain. 2-BBB’s G-Technology empowers sustained delivery of systemically administered therapeutics to the brain with high safety and efficacy profiles. The G-Technology ${ }^{\circ}$ uses PEG and glutathione bioconjugated to active pharmaceutical ingredients loaded liposomal vesicular structures. Polyethylene glycol (PEG) is attached to the liposomes to provide a prolonged circulation time in the blood stream with sustained halflife pharmacokinetic profile. Glutathione is bioconjugated to the PEG molecules to offer targeted safe and effective delivery of the therapeutics across the $\mathrm{BBB}^{[78]}$.

2-BBB has adopted its G-Technology in developing two lead clinical programs targeting multiple indications of brain cancers and neuroinflammatory diseases. 2-BBB's lead product 2B3-101 (glutathione PEGylated liposomal doxorubicin) combines the G-Technology ${ }^{\circledR}$ with the existing chemotherapeutic agent doxorubicin for the possible treatment of brain metastases and glioma. It has completed a Phase I/IIa trial treating patients with various forms of brain cancer. 2-BBB's second promising lead product is 2B3-102. 2B3-102 is a glutathione PEGylated liposomal methylprednisolone. It applies the anti-inflammatory glucocorticoid methylprednisolone to combat acute and chronic neuro-inflammation associated with several CNS 
indications including acute relapses of multiple sclerosis, optic neuritis, neuromyelitis optica and uveitis. 2B3-201 has completed a Phase I clinical trial in healthy volunteers ${ }^{[77]}$.

2-BBB is a clear example of industry-driven research aiming at developing innovative passive targeting technologies to brain and brain metastases. They use triple passive targeting techniques to offer optimal safety and efficacy profiles, Glutathione passively targeting the cancerous intracellular reductive environment, PEGylation and liposomal vesicular carrier system ${ }^{[77]}$.

\section{Nucleic acid}

RNA interference (RNAi) is an endogenous pathway for post-transcriptional silencing of gene expression that is triggered by double-stranded RNA, including endogenous microRNA (miRNA) and synthetic short interfering RNA ${ }^{[79]}$. MicroRNA-122 mediated RNAi brings new prospects ${ }^{[80]}$. Zhang et al ${ }^{[81]}$ miRNA-1258 in $\mathrm{BMBC}$ cells inhibited heparanase which regulates many molecules involved in angiogenesis and metastasis of the tumor.

\section{Advanced physically manipulated systems to disrupt $B B B$}

Another modality of addressing BBB passively is advanced physically manipulated systems that can be tightly mediated by stimuli to treat diseases specifically and with a controlled dosage of drugs. Physical manipulation can be achieved based on ultrasound, electricity, magnetism and photonic emission technologies ${ }^{[82]}$. Davalos et al. ${ }^{[83]}$ applied pulsed electric fields into brain tissue of an animal, to cause temporary disruption of the BBB in a volume of brain tissue near the source of the pulsed electric fields over a specified time interval.

The use of focused ultrasound (FUS) combined with circulating microbubbles is a non-invasive method that increases the permeability of BBTB and improves outcomes of trastuzumab; this technique was used by Park et al. ${ }^{[84]}$ to improve outcomes with trastuzumab in a breast cancer brain metastasis model. Similarly, FUS in combination with microbubbles was able to temporarily disrupt the BBB enhancing the anti-tumor efficacy of the two anti-HER2 agents combination therapy ${ }^{[85]}$. Moreover, reversible disruption of the BBB by bursts of low frequency MRI-guided ultrasound enhances the brain delivery of monoclonal antibody Herceptin (trastuzumab) in mice ${ }^{[86,87]}$.

\section{Active targeting}

Active targeting was proposed for improved targeting efficacy ${ }^{[47]}$. These strategies consist in incorporating affinity molecules or taking advantage of influx transport systems expressed within the BBB/BBTB depending on specific interactions of ligand-receptor and antibody-antigen ${ }^{[47,88,89]}$. Also, several ligands have been studied and utilized to shuttle nanoparticles, antibodies, and drugs across the BBB and into the brain cells $^{[00]}$ [Figure 1]. Supplementary Table 2 summarizes recent studies of actively targeted treatments for BCBM.

\section{Receptor-mediated transcytosis/ligand-based}

An overexpression of receptors or antigens in cancer acts as a potential target to achieve efficient drug uptake via receptor-mediated endocytosis ${ }^{[47]}$. Moreover, receptor-mediated transcytosis (RMT) allows for $\mathrm{BBB}$ transport of various macromolecules after initial binding of a targeting ligand to a receptor expressed on the brain endothelial cells ${ }^{[91]}$. Tumor-targeting ligands such as peptides and antibodies may effectively aid certain cytotoxic agents (either biological or synthetic) to deliver to the tumor cells, thereby improving therapeutic efficacy while limiting the exposure of normal tissues to the cytotoxic agents ${ }^{[92]}$. The transferrin receptor, the low-density lipoprotein receptor-related protein 1 (LRP-1), the insulin receptor and the nicotinic acetylcholine receptors are examples of receptor expression on the BBB. 
AngioChem ${ }^{\mathrm{TM}}$ Inc. developed a series of aprotinin polypeptides (Angiopeps) ${ }^{[93]}$. Angiopep-2, a 19-aminoacid peptide, is one of the promising vectors designed to target the LRP-1 receptor, to mediate transcytosis across the BBB. It is derived from the human Kunitz domain ${ }^{[94]}$. Angiopep-2 can facilitate brain-targeted drug delivery through LRP-1-mediated transcytosis. Regina et al. ${ }^{[95]}$ demonstrated that a conjugate between angiopep-2 and an anti-HER2 mAb results in a new chemical entity, ANG4043. ANG4043 retains in-vitro binding affinity for the HER2 receptor and antiproliferative potency against BCBM rat model. This study showed increased uptake in brain endothelial cells and enhanced BBB permeability compared to poor brain penetration of anti-HER2 mAb alone. Similarly, Thomas et al. ${ }^{[96]}$ showed that ANG1005 shows significantly improved delivery to brain and brain metastases of breast cancer compared to free paclitaxel in mice bearing BCBM.

On the other hand, Orthmann et al. ${ }^{[97]}$ formulated rigid and fluid liposomes entrapping Mitoxantrone and equipped with a 19-mer angiopeptide as a ligand. Angiopeptide bearing fluid liposomes showed in vitro the highest cellular uptake and transcytosis. They were significantly better than the corresponding ligandfree fluid liposomes and ligand-bearing rigid vesicles however; the improvement was mainly depending on liposomal fluidity while the targeting contributed only to a minor degree. In 2016, Orthmann et al. ${ }^{[98]}$ encapsulated oxaliplatin ( $\mathrm{OxP})$ in liposomes then bound angiopep-2 to the vesicular surface. They determined that the newly developed OxP liposomes significantly improved the treatment of subcutaneously and intracerebrally growing breast cancer, but the targeted angiopep-equipped liposomes showed no superior effect in vivo.

Melanotransferrin (hMTf) is another target to the LRP-1 receptor, which was shown to deliver doxorubicin across $\mathrm{BBB}^{[99,100]}$. In order to overcome the trastuzumab inability to cross the $\mathrm{BBB}$ and treat brain metastases of HER2+ breast cancer, BT2111, a novel bioconjugate of trastuzumab was developed by BiOasis Inc., on the hMTf (p97) Transcend ${ }^{\mathrm{TM}}$ vector platform ${ }^{[101]}$. This platform is an example of an actively targeted immunotherapy, aiding in enhancing the immunotherapy targetability, ability to cross the BB and clinical efficacy via the use of the targeting moiety hMTf.

Another complex process involves binding to a primary, tumor-specific receptor activating endocytosis; using "Tumor-penetrating peptide" ${ }^{,[102]}$. The prototypic peptide of this class, iRGD (CRGDKGPDC), contains the integrin-binding RGD motif ${ }^{[103]}$. The integrin-binding RGD sequence motif binds to $\alpha v \beta 3$ and $\alpha v \beta 5$ integrins, which are specifically expressed in tumor endothelial cells ${ }^{[102]}$. Hamilton et al ${ }^{[104]}$ demonstrated that a single dose of iRGD had a significant effect on metastatic tumor progression and nonproliferative cancer cell retention when applied early in course of tumor development. Proteolytically processed iRGD also exerts anti-metastatic activity by binding to neuropilin-1 and activating an endocytic bulk transport pathway through tumor tissue. The iRGB platform offers an innovative dual targeting tool to target the tumor portion via the integrins and the RGB motif ${ }^{[103]}$.

\section{Carrier-mediated transcytosis}

Naturally, carrier-mediated transcytosis (CMT) enables spontaneous internalization of small biomolecules ${ }^{[105,106]}$. CMT takes advantage of the immunological surveillance system of the brain, using circulating phagocytic cells such as monocytes or macrophages as Trojan horse to deliver drug molecules into the brain ${ }^{[107]}$. Such cells have a tendency to endocytose colloidal materials, for example, nano or microparticles, liposomes, and subsequent exocytosis to release drug and/or colloidal materials to external media ${ }^{[108]}$. Fidler and colleagues provided evidence that macrophages of blood monocyte origin can infiltrate experimental brain metastases while the BBB is intact.

In 2012, Choi et al ${ }^{[109]}$ reported the first successful demonstration of the active delivery, using macrophages, of nanoparticles to brain metastases. Activated macrophages not only cross the BBB but they envelop the 
metastatic cells delivering the loaded nanoparticle to less than a cell width away from the nearest metastatic cell ${ }^{[109]}$.

\section{Cell penetrating peptides}

Cell-penetrating peptides (CPP), that show great capacity in $\mathrm{BBB}$ transport, have the ability to transport protein or peptides into cells in a nonspecific, receptor-independent manner and non-immunogenic when compared with antibodies ${ }^{[110]}$. Considering their smaller size (up to 30 amino acids in length), cationic and/ or amphipathic CPPs have a greater potential to penetrate the BBB than other transport systems. Short peptides, as targeted drug delivery vehicles, appear to have some advantages owing to their small size, efficient tissue penetrability, and minimal toxicity and immunogenicity.

The first CPP, trans-activator of transcription (TAT), derived from human immunodeficiency virus-1, can be efficiently taken up from the surrounding media ${ }^{[111]}$. Morshed et al. ${ }^{[12]}$ used PEGylated gold nanoparticle conjugated to TAT peptide as well as doxorubicin. This formulation offered extensive accumulation of particles throughout diffuse intracranial metastatic microsatellites. Moreover, it was shown to destabilize a brain capillary monolayer increasing its permeability. Fu et al. ${ }^{[13]}$ combined brain metastatic breast carcinoma cell (231-BR)-binding peptide BRBP1, a cell penetrating peptide TAT, and a proapoptotic peptide KLA. The composite selectively homed to the tumors in vivo where it induced cellular apoptosis without significant toxicity on non-tumor tissues. Angiopep-mediated targeting also can be considered as one of the most promising ways to reach the CNS for the treatment of brain cancer or brain metastases ${ }^{[114]}$.

\section{Conclusion and future directions}

There is a global need for effective and safe pharmaceutical chemotherapeutic agents that have the potential to target tumors like BCBM.

Conventional chemotherapy, radiation therapy and immunotherapy offers promising options for treating brain metastases, which is traditionally treated with surgery.

In 2005, the chemotherapy temozolomide (Temodar ${ }^{\circ}$ ) was approved to treat glioblastoma (GBM) patients. However, over 50\% of GBM tumors generate a DNA repair protein (methylguanine methyltransferase) that effectively combats and neutralizes temozolomide chemotherapy. There are two FDA approved immunotherapies for brain and nervous system cancers and metastases. Bevacizumab (Avastin ${ }^{\circ}$ ) and Dinutuximab (Unituxin ${ }^{\circ}$ ). Several other immunotherapies are being used to treat different types of brain cancers in clinical trials. However, there are no FDA-approved systemic treatments specific to BCBM to date ${ }^{[41]}$. Improved insights into the microenvironment and metastatic cascade processes have resulted in the development of several novel chemotherapeutic and immunotherapeutic drugs and strategies ${ }^{[35,36,38,39,54]}$. Such novel chemotherapeutic and immunotherapeutic strategies adopt active and passive targeting strategies to enhance the clinical treatment effects.

Surveying the published research papers and patents also revealed a major drawback in the methodology design, which is the lack of standardization of efficacy and safety profiles. Various sophisticated nanosystems and conjugates have been studied to either actively or passively target BCBM. However, most of these complex delivery systems fail to reach the market due to high cost, instability and difficulty of scaling up. In addition, majority of the reagents used in the formulation of such novel therapeutic systems to improve the stability; are not included in the FDA approved inactive ingredient database. The main question of this review is to query the preferential advantage of either active or passive targeting in combating brain metastases of breast cancer. Such question is a major ongoing debate as revealed by the recent patents and papers portfolio. 
Passive targeting could be a cheaper, long-term effective and safe technique to target tumor cells without triggering resistance for active targeting moieties. Numerous significant downsides in active tumor-targeted drug delivery were identified ${ }^{[115-120]}$. Interestingly, passive targeting can be tailored and designed via simple ideologies and techniques such as simple PEGylation, size modifications or even shape customization. It was shown that slender shape and particles modifications such as drifting from the conventional spherical particulate structures, can yield high effectual passive targeting ${ }^{[121]}$.

Active targeting shows significant results in combating cancer initially with long-term degraded efficacy profile, most potentially attributed to multidrug resistance ${ }^{[119]}$. Additionally, the marginally augmented efficacy of active targeting strategies does not substantiate the extremely higher cost, difficulty in clinical application and the high complexity level of the carrier system ${ }^{[5,78,115-120,122,123]}$. Interestingly, passive targeting can be tailored and designed via simple ideologies and techniques such as simple PEGylation, size modifications or even shape customization.

Multiple researchers suggested the preferential long-term advantage of passive targeting over active targeting such as Lammers et al. ${ }^{[122]}$ and Rosenblum et al. ${ }^{[123]}$. Such researches highlighted the need for future realistic and scalable efforts to address some of the conceptual drawbacks of drug targeting to tumors such as resistance, and that strategies should be developed to overcome these deficiencies. Such research presented evidence that passive targeting yield very comparable results to active targeting on the short term and superior results on the long term ${ }^{[54,78,115-120,122,123]}$.

Some of the main drawbacks of engineered nanotechnologies, either active or passive, are the poor robust and cost-effective toolbox to characterize nanomaterials and to quantify exposure in test systems (dosimetry), lack of standard techniques and methods for test, hazard, and risk assessment strategies and lack of specific safety assessment strategies in evaluating nanotoxicities. However, passive targeting offers less problematic strategy compared to active targeting engineered nanotechnologies in terms of nano-safety and its quantitative characterization ${ }^{[124]}$.

Passive targeting could provide an applied direction for the advance of novel management tackles and therapeutics for brain metastases of breast cancer for researchers worldwide, paving the road to affordable, scalable, stable, efficient and safe management strategies. Such observations were evident from the multiple passive targeting products under Phase II and Phase III clinical trials from multiple pharmaceutical firms such as Nektar Therapeutics, BiOasis Inc. and 2-BBB. Such companies have invested heavily into the development of new formulations that combat brain barriers, targeting the brain and accumulates in tumor tissue rather than normal cells, to enhance the nano-safety profiles to chemotherapeutics. This may not be only attributed to their need to expand their portfolio to increase their commercial value for possible products buyouts and licensing by big pharmaceutical firms, but the safety and efficacy potential of such passively targeted nanotherapeutics in the treatment strategies of brain metastases of breast cancer compared to current conventional modalities and research-driven active-targeting initiatives.

Such conclusions have driven the industry to invest heavily on passive targeting compared to active targeting. A major focus by industry is the successful transformation of such novel technologies from bench to bedside with reasonable cost, scale-up abilities and formulation robustness and reproducibility, which are achievable via passive targeting technologies compared to active targeting. Active targeting ligand post-insertion and labeling techniques need to be extensively researched for ease of application for active targeting to gain industrial momentum. Conclusively, patents and products under development should focus on simple passively targeted bioconjugate structures, which are easily synthesized with high yield, reduced cost and high stability profile of the final formulation. 


\section{DECLARATIONS}

\section{Authors' contributions}

Manuscript writing: Kamal NH, El-Amrawy F, Ali HA, Nounou MI

Manuscript proofing and revisions: Edafiogho I, Nounou MI

Design: Nounou MI

\section{Availability of data and materials}

Not applicable.

\section{Financial support and sponsorship}

None.

\section{Conflicts of interest}

All authors declared that there are no conflicts of interest.

\section{Ethical approval and consent to participate}

Not applicable.

\section{Consent for publication}

Not applicable.

\section{Copyright}

(c) The Author(s) 2019.

\section{REFERENCES}

1. Lin NU. Breast cancer brain metastases: new directions in systemic therapy. ecancermedicalscience 2013;7:307.

2. Palmieri D, Smith QR, Lockman PR, Bronder J, Gril B, et al. Brain metastases of breast cancer. Breast Dis 2006;26:139-47.

3. Yeh RH, Yu JC, Chu CH, Ho CL, Kao HW, et al. Distinct MR imaging features of triple-negative breast cancer with brain metastasis. J Neuroimaging 2015;25:474-81.

4. Steeg PS, Camphausen KA, Smith QR. Brain metastases as preventive and therapeutic targets. Nat Rev Cancer 2011;11:352-63.

5. Lin NU, Claus E, Sohl J, Razzak AR, Arnaout A, et al. Sites of distant recurrence and clinical outcomes in patients with metastatic triplenegative breast cancer: high incidence of central nervous system metastases. Cancer 2008;113:2638-45.

6. Aversa C, Rossi V, Geuna E, Martinello R, Milani A, et al. Metastatic breast cancer subtypes and central nervous system metastases. Breast 2014;23:623-8.

7. Clayton AJ, Danson S, Jolly S, Ryder WDJ, Burt PA, et al. Incidence of cerebral metastases in patients treated with trastuzumab for metastatic breast cancer. Br J Cancer 2004;91:639-43.

8. Witzel I, Oliveira-Ferrer L, Pantel K, Muller V, Wikman H. Breast cancer brain metastases: biology and new clinical perspectives. Breast Cancer Res 2016;18:8

9. Lin X, DeAngelis LM. Treatment of brain metastases. J Clin Oncol 2015;33:3475-84.

10. Kotecki N, Lefranc F, Devriendt D, Awada A. Therapy of breast cancer brain metastases: challenges, emerging treatments and perspectives. Ther Adv Med Oncol 2018;10:1758835918780312.

11. Adkins CE, Mohammad AS, Terrell-Hall TB, Dolan EL, Shah N, et al. Characterization of passive permeability at the blood-tumor barrier in five preclinical models of brain metastases of breast cancer. Clin Exp Metastasis 2016;33:373-83.

12. O'Sullivan CC, Davarpanah NN, Abraham J, Bates SE. Current challenges in the management of breast cancer brain metastases. Semin Oncol 2017;44:85-100.

13. Ballabh P, Braun A, Nedergaard M. The blood-brain barrier: an overview: structure, regulation, and clinical implications. Neurobiol Dis 2004;16:1-13.

14. Cook LJ, Freedman J. Brain tumors. New York: The Rosen Publishing Group; 2011.

15. Dauchy S, Miller F, Couraud PO, Weaver RJ, Weksler B, et al. Expression and transcriptional regulation of ABC transporters and cytochromes P450 in hCMEC/D3 human cerebral microvascular endothelial cells. Biochem Pharmacol 2009;77:897-909.

16. Abbott NJ, Patabendige AA, Dolman DE, Yusof SR, Begley DJ. Structure and function of the blood-brain barrier. Neurobiol Dis 2010;37:13-25.

17. Van Tellingen O, Yetkin-Arik B, De Gooijer MC, Wesseling P, Wurdinger T, et al. Overcoming the blood-brain tumor barrier for effective glioblastoma treatment. Drug Resist Updat 2015;19:1-12.

18. Dong X. Current strategies for brain drug delivery. Theranostics 2018;8:1481-93. 
19. Rip J, Schenk GJ, de Boer AG. Differential receptor-mediated drug targeting to the diseased brain. Expert Opin Drug Deliv 2009;6:227-37.

20. Abbott NJ, Friedman A. Overview and introduction: The blood-brain barrier in health and disease. Epilepsia 2012;53:1-6.

21. Banks WA. From blood-brain barrier to blood-brain interface: new opportunities for CNS drug delivery. Nat Rev Drug Discov 2016;15:275-92.

22. Anderson A, Choy C, Neman J, Duenas MJ, Jandial R, et al. Metastatic breast cancer to the brain: a clinical primer for translational investigation. Madame Curie Bioscience Database [Internet]. Austin (TX): Landes Bioscience; 2013.

23. Weber GF. Molecular mechanisms of metastasis. Cancer Lett 2008;270:181-90.

24. Banys-Paluchowski M, Krawczyk N, Meier-Stiegen F, Fehm T. Circulating tumor cells in breast cancer - current status and perspectives. Crit Rev Oncol Hematol 2016;97:22-9.

25. Prieto-Vila M, Takahashi RU, Usuba W, Kohama I, Ochiya T. Drug resistance driven by cancer stem cells and their niche. Int J Mol Sci 2017; 18:E2574.

26. Rahmathulla G, Toms SA, Weil RJ. The molecular biology of brain metastasis. J Oncol 2012;2012:723541.

27. Seoane J, De Mattos-Arruda L. Brain metastasis: new opportunities to tackle therapeutic resistance. Mol Oncol 2014;8:1120-31.

28. Jin X, Mu P. Targeting breast cancer metastasis. Breast Cancer (Auckl) 2015;9:23-34 .

29. Bos PD, Zhang XH, Nadal C, Shu W, Gomis RR, et al. Genes that mediate breast cancer metastasis to the brain. Nature 2009;459:1005-9.

30. Leek RD, Harris AL, Lewis CE. Cytokine networks in solid human tumors: regulation of angiogenesis. J Leukoc Biol 1994;56:423-35.

31. Cheng X, Hung MC. Breast cancer brain metastases. Cancer Metastasis Rev 2007;26:635-43.

32. Lee BC, Lee TH, Avraham S, Avraham HK. Involvement of the chemokine receptor CXCR4 and its ligand stromal cell-derived factor 1alpha in breast cancer cell migration through human brain microvascular endothelial cells. Mol Cancer Res 2004;2:327-38.

33. Avraham HK, Jiang S, Fu Y, Nakshatri H, Ovadia H, et al. Angiopoietin-2 mediates blood-brain barrier impairment and colonization of triple-negative breast cancer cells in brain. J Pathol 2014;232:369-81.

34. Ahmad SA, Liu W, Jung YD, Fan F, Wilson M, et al. The effects of angiopoietin-1 and -2 on tumor growth and angiogenesis in human colon cancer. Cancer Res 2001;61:1255-9.

35. Valiente M, Obenauf AC, Jin X, Chen Q, Zhang XHF, et al. Serpins promote cancer cell survival and vascular co-option in brain metastasis. Cell 2014;156:1002-16.

36. Becker A, Thakur BK, Weiss JM, Kim HS, Peinado H, et al. Extracellular vesicles in cancer: cell-to-cell mediators of metastasis. Cancer cell 2016;30:836-48.

37. Lin NU, Bellon JR, Winer EP. CNS metastases in breast cancer. J Clin Oncol 2004;22:3608-17.

38. Lockman PR, Mittapalli RK, Taskar KS, Rudraraju V, Gril B, et al. Heterogeneous blood-tumor barrier permeability determines drug efficacy in experimental brain metastases of breast cancer. Clin Cancer Res 2010;16:5664-78.

39. Villanueva MT. Drug therapy: smuggling trastuzumab into the brain. Nat Rev Clin Oncol 2013;10:669.

40. Yonemori K, Tsuta K, Ono M, Shimizu C, Hirakawa A, et al. Disruption of the blood brain barrier by brain metastases of triple-negative and basal-type breast cancer but not HER2/neu-positive breast cancer. Cancer 2010;116:302-8.

41. Brosnan EM, Anders CK. Understanding patterns of brain metastasis in breast cancer and designing rational therapeutic strategies. Ann Transl Med 2018;6:163.

42. ELAmrawy F, Othman AA, Adkins C, Helmy A, Nounou MI. Tailored nanocarriers and bioconjugates for combating glioblastoma and other brain tumors. J Cancer Metastasis Treat 2016;2:112-22.

43. Kazantsev AG, Outeiro TF. Drug discovery for CNS disorders: from bench to bedside. CNS Neurol Disord Drug Targets 2010;9:668.

44. Gabathuler R. Approaches to transport therapeutic drugs across the blood-brain barrier to treat brain diseases. Neurobiol Dis 2010;37:48-57.

45. Pardridge WM. Drug delivery to the brain. J Cereb Blood Flow Metab 1997;17:713-31.

46. Barar J, Rafi MA, Pourseif MM, Omidi Y. Blood-brain barrier transport machineries and targeted therapy of brain diseases. BioImpacts 2016;6:225-48.

47. Dhankhar R, Vyas SP, Jain AK, Arora S, Rath G, et al. Advances in novel drug delivery strategies for breast cancer therapy. Artif Cells Blood Substit Immobil Biotechnol 2010;38:230-49.

48. Matsumura Y, Maeda H. A new concept for macromolecular therapeutics in cancer chemotherapy: mechanism of tumoritropic accumulation of proteins and the antitumor agent smancs. Cancer Res 1986;46:6387-92.

49. Kobayashi H, Turkbey B, Watanabe R, Choyke PL. Cancer drug delivery: considerations in the rational design of nanosized bioconjugates. Bioconjug Chem 2014;25:2093-100.

50. Singh R, Lillard JW Jr. Nanoparticle-based targeted drug delivery. Experimental and molecular pathology 2009;86:215-23.

51. Blanco E, Shen H, Ferrari M. Principles of nanoparticle design for overcoming biological barriers to drug delivery. Nat Biotechnol 2015;33:941-51.

52. Liechty WB, Peppas NA. Expert opinion: responsive polymer nanoparticles in cancer therapy. Eur J Pharm Biopharm 2012;80:241-6.

53. Davis ME, Chen ZG, Shin DM. Nanoparticle therapeutics: an emerging treatment modality for cancer. Nat Rev Drug Discov 2008;7:771-82.

54. Lammers T, Kiessling F, Hennink WE, Storm G. Drug targeting to tumors: principles, pitfalls and (pre-) clinical progress. J Control Release 2012;161:175-87.

55. Din FU, Aman W, Ullah I, Qureshi OS, Mustapha O, et al. Effective use of nanocarriers as drug delivery systems for the treatment of selected tumors. Int J Nanomedicine 2017;12:7291-309.

56. Weissenbock A, Wirth M, Gabor F. WGA-grafted PLGA-nanospheres: preparation and association with Caco-2 single cells. J Control Release 2004;99:383-92.

57. Wesselinova D. Current major cancer targets for nanoparticle systems. Curr Cancer Drug Targets 2011;11:164-83.

58. De Jong WH, Borm PJ. Drug delivery and nanoparticles:applications and hazards. Int J Nanomedicine 2008;3:133-49.

59. Perche F, Torchilin VP. Recent trends in multifunctional liposomal nanocarriers for enhanced tumor targeting. J Drug Deliv 
2013;2013:705265.

60. Mohammad AS, Griffith JI, Adkins CE, Shah N, Sechrest E, et al. Liposomal irinotecan accumulates in metastatic lesions, crosses the blood-tumor barrier (BTB), and prolongs survival in an experimental model of brain metastases of triple negative breast cancer. Pharm Res 2018;35:31.

61. Hamidi M, Azadi A, Rafiei P. Pharmacokinetic consequences of pegylation. Drug Delivery 2006;13:399-409.

62. Salmaso S, Caliceti P. Stealth properties to improve therapeutic efficacy of drug nanocarriers. J Drug Deliv 2013;2013:374252.

63. Vail DM, Amantea MA, Colbern GT, Martin FJ, Hilger RA, et al. Pegylated liposomal doxorubicin: proof of principle using preclinical animal models and pharmacokinetic studies. Semin Oncol 2004;31:16-35.

64. Yokoyama M, Miyauchi M, Yamada N, Okano T, Sakurai Y, et al. Characterization and anticancer activity of the micelle-forming polymeric anticancer drug adriamycin-conjugated poly(ethylene glycol)-poly(aspartic acid) block copolymer. Cancer Res 1990;50:1693-700.

65. James ND, Coker RJ, Tomlinson D, Harris JR, Gompels M, et al. Liposomal doxorubicin (Doxil): an effective new treatment for Kaposi's sarcoma in AIDS. Clin Oncol (R Coll Radiol) 1994;6:294-6.

66. Muggia FM. Doxil in breast cancer. J Clin Oncol 1998;16:811-2.

67. Porche DJ. Liposomal doxorubicin (Doxil). J Assoc Nurses AIDS Care 1996;7:55-9.

68. Anders CK, Adamo B, Karginova O, Deal AM, Rawal S, et al. Pharmacokinetics and efficacy of PEGylated liposomal doxorubicin in an intracranial model of breast cancer. PLoS One 2013;8:e61359.

69. Zhao X, Bentley MD, Ren Z, Viegas TX. Multi-arm polymer prodrugs. In: Therapeutics N, editor. The United States Patent and Trademark Office. US: Nektar Therapeutics; 2013.

70. Zhang W. Method for preparing a polymer conjugate. USA: Nektar therapeutics; 2013.

71. Minamitani EL, Zappe H, Bossard MJ, Roczniak SO, Liu X. Polymer conjugates of kiss1 peptides. USA: Nektar therapeutics; 2011.

72. Hoch U, Eldon MA, Leung ACF. Treatment of patients suffering from cancer. In: Therapeutics N, editor. The United States Patent and Trademark Office. US: Nektar Therapeutics; 2013.

73. Fishburn CS, Lechuga-Ballesteros D, Viegas T, Kuo M, Song Y, et al. Chemically modified small molecules. In: Therapeutics N, editor. The United States Patent and Trademark Office. US: Nektar Therapeutics; 2011.

74. Eldon MA, Harite SS, Barker TL. Compositions and methods for achieving sustained therapeutic drug concentrations in a subject. In: Therapeutics N, editor. The United States Patent and Trademark Office. US: Nektar Therapeutics; 2011.

75. Chen YC, Chiang CF, Chen LF, Liang PC, Hsieh WY, et al. Polymersomes conjugated with des-octanoyl ghrelin and folate as a BBBpenetrating cancer cell-targeting delivery system. Biomaterials 2014;35:4066-81.

76. Adkins CE, Nounou MI, Hye T, Mohammad AS, Terrell-Hall T, et al. NKTR-102 Efficacy versus irinotecan in a mouse model of brain metastases of breast cancer. BMC Cancer 2015;15:685.

77. Gaillard PJ. 2-BBB Products' Pipline. Leiden Bio Science Park, The Netherlands: 2-BBB Medicines BV; 2018.

78. Geldenhuys W, Wehrung D, Groshev A, Hirani A, Sutariya V. Brain-targeted delivery of doxorubicin using glutathione-coated nanoparticles for brain cancers. Pharm Dev Technol 2015;20:497-506.

79. Kanasty R, Dorkin JR, Vegas A, Anderson D. Delivery materials for siRNA therapeutics. Nat Mater 2013;12:967-77.

80. Wang G, Jia T, Xu X, Chang L, Zhang R, et al. Novel miR-122 delivery system based on MS2 virus like particle surface displaying cellpenetrating peptide TAT for hepatocellular carcinoma. Oncotarget 2016;7:59402-16.

81. Zhang L, Sullivan PS, Goodman JC, Gunaratne PH, Marchetti D. MicroRNA-1258 suppresses breast cancer brain metastasis by targeting heparanase. Cancer Res 2011;71:645-54.

82. Rodriguez-Devora JI, Ambure S, Shi Z-D, Yuan Y, Sun W, et al. Physically facilitating drug-delivery systems. Therapeutic delivery 2012;3:125-39.

83. Davalos RV, Rossmeisl JH, Garcia PA. Acute blood-brain barrier disruption using electrical energy based therapy. Virginia Tech Intellectual Properties, Inc.; 2014.

84. Park EJ, Zhang YZ, Vykhodtseva N, McDannold N. Ultrasound-mediated blood-brain/blood-tumor barrier disruption improves outcomes with trastuzumab in a breast cancer brain metastasis model. J Control Release 2012;163:277-84.

85. Kobus T, Zervantonakis IK, Zhang Y, McDannold NJ. Growth inhibition in a brain metastasis model by antibody delivery using focused ultrasound-mediated blood-brain barrier disruption. J Control Release 2016;238:281-8.

86. Kinoshita M, McDannold N, Jolesz FA, Hynynen K. Noninvasive localized delivery of Herceptin to the mouse brain by MRI-guided focused ultrasound-induced blood-brain barrier disruption. Proceedings of the National Academy of Sciences 2006;103:11719-23.

87. Kinoshita M, McDannold N, Jolesz FA, Hynynen K. Noninvasive localized delivery of Herceptin to the mouse brain by MRI-guided focused ultrasound-induced blood-brain barrier disruption. Proc Natl Acad Sci U S A 2006;103:11719-23.

88. Nobs L, Buchegger F, Gurny R, Allemann E. Poly(lactic acid) nanoparticles labeled with biologically active Neutravidin for active targeting. Eur J Pharm Biopharm 2004;58:483-90.

89. Prinzen L, Miserus RJ, Dirksen A, Hackeng TM, Deckers N, et al. Optical and magnetic resonance imaging of cell death and platelet activation using annexin a5-functionalized quantum dots. Nano Lett 2007;7:93-100.

90. Wang YY, Lui PC, Li JY. Receptor-mediated therapeutic transport across the blood-brain barrier. Immunotherapy 2009;1:983-93.

91. Jones AR, Shusta EV. Blood-brain barrier transport of therapeutics via receptor-mediation. Pharm Res 2007;24:1759-71.

92. Lin NU. Targeted therapies in brain metastases. Curr Treat Options Neurol 2014;16:276.

93. Beliveau R. Method for transporting a compound across the blood-brain barrier. AngioChem Inc.; 2003.

94. Regina A, Demeule M, Che C, Lavallee I, Poirier J, et al. Antitumour activity of ANG1005, a conjugate between paclitaxel and the new brain delivery vector Angiopep-2. Br J Pharmacol 2008;155:185-97.

95. Regina A, Demeule M, Tripathy S, Lord-Dufour S, Currie JC, et al. ANG4043, a novel brain-penetrant peptide-mAb conjugate, is efficacious against HER2-positive intracranial tumors in mice. Mol Cancer Ther 2015;14:129-40.

96. Thomas FC, Taskar K, Rudraraju V, Goda S, Thorsheim HR, et al. Uptake of ANG1005, a novel paclitaxel derivative, through the blood- 
brain barrier into brain and experimental brain metastases of breast cancer. Pharm Res 2009;26:2486-94.

97. Orthmann A, Zeisig R, Suss R, Lorenz D, Lemm M, et al. Treatment of experimental brain metastasis with MTO-liposomes: impact of fluidity and LRP-targeting on the therapeutic result. Pharm Res 2012;29:1949-59.

98. Orthmann A, Peiker L, Fichtner I, Hoffmann A, Hilger RA, et al. Improved treatment of MT-3 breast cancer and brain metastases in a mouse xenograft by LRP-targeted oxaliplatin liposomes. J Biomed Nanotechnol 2016;12:56-68.

99. Demeule M, Poirier J, Jodoin J, Bertrand Y, Desrosiers RR, et al. High transcytosis of melanotransferrin (P97) across the blood-brain barrier. J Neurochem 2002;83:924-33.

100. Dorries R. The role of T-cell-mediated mechanisms in virus infections of the nervous system. Curr Top Microbiol Immunol 2001;253:219-45.

101. Nounou MI, Adkins CE, Rubinchik E, Terrell-Hall TB, Afroz M, et al. Anti-cancer antibody trastuzumab-melanotransferrin conjugate (BT2111) for the treatment of metastatic HER2+ breast cancer tumors in the brain: an in-vivo study. Pharm Res 2016;33:2930-42.

102. Ruoslahti E. Tumor penetrating peptides for improved drug delivery. Advanced Drug Delivery Reviews 2017;110-111:3-12.

103. Teesalu T, Sugahara KN, Ruoslahti E. Tumor-penetrating peptides. Front Oncol 2013;3:216.

104. Hamilton AM, Aidoudi-Ahmed S, Sharma S, Kotamraju VR, Foster PJ, et al. Nanoparticles coated with the tumor-penetrating peptide iRGD reduce experimental breast cancer metastasis in the brain. J Mol Med (Berl) 2015;93:991-1001.

105. Pardridge WM. Blood-brain barrier carrier-mediated transport and brain metabolism of amino acids. Neurochem Res 1998;23:635-44.

106. Tsuji A. Small molecular drug transfer across the blood-brain barrier via carrier-mediated transport systems. NeuroRx 2005;2:54-62.

107. Chiou B, Neal EH, Bowman AB, Lippmann ES, Simpson IA, et al. Pharmaceutical iron formulations do not cross a model of the human blood-brain barrier. PloS One 2018;13:e0198775.

108. Lameijer MA, Tang J, Nahrendorf M, Beelen RHJ, Mulder WJM. Monocytes and macrophages as nanomedicinal targets for improved diagnosis and treatment of disease. Expert Rev Mol Diagn 2013;13:567-80.

109. Choi M-R, Bardhan R, Stanton-Maxey KJ, Badve S, Nakshatri H, et al. Delivery of nanoparticles to brain metastases of breast cancer using a cellular Trojan horse. Cancer nanotechnol 2012;3:47-54.

110. Stalmans S, Bracke N, Wynendaele E, Gevaert B, Peremans K, et al. Cell-penetrating peptides selectively cross the blood-brain barrier in vivo. PLoS One 2015;10:e139652.

111. Mae M, Langel U. Cell-penetrating peptides as vectors for peptide, protein and oligonucleotide delivery. Curr Opin Pharmacol 2006;6:509-14.

112. Morshed RA, Muroski ME, Dai Q, Wegscheid ML, Auffinger B, et al. Cell-penetrating peptide-modified gold nanoparticles for the delivery of doxorubicin to brain metastatic breast cancer. Mol Pharm 2016;13:1843-54.

113. Fu B, Long W, Zhang Y, Zhang A, Miao F, et al. Enhanced antitumor effects of the BRBP1 compound peptide BRBP1-TAT-KLA on human brain metastatic breast cancer. Sci Rep 2015;5:8029.

114. Meyers JD, Doane T, Burda C, Basilion JP. Nanoparticles for imaging and treating brain cancer. Nanomedicine (Lond) 2013;8:123-43.

115. Kunjachan S, Pola R, Gremse F, Theek B, Ehling J, et al. Passive versus active tumor targeting using RGD- and NGR-modified polymeric nanomedicines. Nano Lett 2014;14:972-81.

116. Golombek SK, May JN, Theek B, Appold L, Drude N, et al. Tumor targeting via EPR: strategies to enhance patient responses. Adv Drug Deliv Rev 2018;130:17-38.

117. Baetke SC, Lammers T, Kiessling F. Applications of nanoparticles for diagnosis and therapy of cancer. Br J Radiol 2015;88:20150207.

118. Kiessling F, Mertens ME, Grimm J, Lammers T. Nanoparticles for imaging: top or flop? Radiology 2014;273:10-28.

119. Kunjachan S, Rychlik B, Storm G, Kiessling F, Lammers T. Multidrug resistance: physiological principles and nanomedical solutions. Adv Drug Deliv Rev 2013;65:1852-65.

120. Lammers T, Rizzo LY, Storm G, Kiessling F. Personalized nanomedicine. Clin Cancer Res 2012;18:4889-94.

121. Devarajan PV, Jindal AB, Patil RR, Mulla F, Gaikwad RV, et al. Particle shape: a new design parameter for passive targeting in splenotropic drug delivery. J Pharm Sci 2010;99:2576-81.

122. Lammers T, Hennink WE, Storm G. Tumour-targeted nanomedicines: principles and practice. Br J Cancer 2008;99:392-7.

123. Rosenblum D, Joshi N, Tao W, Karp JM, Peer D. Progress and challenges towards targeted delivery of cancer therapeutics. Nat Commun 2018;9:1410.

124. Siegrist S, Corek E, Detampel P, Sandstrom J, Wick P, et al. Preclinical hazard evaluation strategy for nanomedicines. Nanotoxicology 2018;1-27. 